\title{
La vieillesse en effigie : figures hyperréalistes et mise en mouvement des représentations collectives
}

Effigies of Old Age: When Hyper-Realistic Figures Renew Collective

Representations

Julie Sermon

\section{OpenEdition}

Journals

Édition électronique

URL : https://journals.openedition.org/recherchestravaux/747

DOI : 10.4000/recherchestravaux.747

ISSN : 1969-6434

Éditeur

UGA Éditions/Université Grenoble Alpes

Édition imprimée

Date de publication : 28 mai 2015

Pagination : $133-147$

ISBN : 978-2-84310-298-1

ISSN : 0151-1874

\section{Référence électronique}

Julie Sermon, « La vieillesse en effigie : figures hyperréalistes et mise en mouvement des

représentations collectives », Recherches \& Travaux [En ligne], 86 | 2015, mis en ligne le 28 novembre

2016, consulté le 29 octobre 2021. URL : http://journals.openedition.org/recherchestravaux/747

DOI : https://doi.org/10.4000/recherchestravaux.747 
Julie Sermon

Université Lumière Lyon 2

Laboratoire Passages XX-XXI

\section{La vieillesse en effigie : figures hyperréalistes et mise en mouvement des représentations collectives}

À la différence du vieillissement, "phénomène biologique aux implications nombreuses, tant au niveau individuel (somatique, psychique) qu'au niveau collectif (démographique, économique, politique) », la vieillesse n'est pas "une donnée, un fait naturel, mais une construction historique et culturelle $»^{\mathrm{I}}$ : un ensemble de représentations - images, valeurs, croyances - qu'une société attache à ses «vieux» et qui, partant, déterminent la place et les pouvoirs qu'elle leur accorde dans l'économie, concrète et symbolique, des âges de la vie. On comprend, dans cette perspective, que la façon qu'ont les artistes de jouer (avec) la vieillesse endosse un rôle d'importance : par ce qu'ils en donnent à voir, entendre, percevoir et penser, ils peuvent aussi bien contribuer à asseoir des stéréotypes qu'à faire évoluer les mentalités, en questionnant les lieux communs ou en affrontant les tabous qui structurent nos imaginaires.

Ce qui m'intéressera dans le travail des artistes marionnettistes que je propose d'analyser ici - à savoir, d'une part, le cycle de création baptisé Les Vieilles, que Julika Mayer (compagnie Là Où) a développé de 2007 à 20II, et d'autre part, le spectacle Silence (2013), créé et interprété par Isabelle Darras et Julie Tenret (compagnie Night Shop Théâtre) - c'est que le trouble et le décalage auxquels ces spectacles soumettent nos représentations de la vieillesse passent

I. J. Foucart, "La vieillesse : une construction sociale», Vieillesse et exclusion sociale, Pensée plurielle, $\mathrm{n}^{\circ}$ 6, 2003/2, Paris, De Boeck Supérieur, p. I3 (article disponible en ligne à l'adresse suivante : <http://www.cairn.info/revue-pensee-plurielle-2003-2-page-7.htm>). 
par la mise en jeu de marionnettes hyperréalistes. Après avoir rappelé quels sont les enjeux techniques, esthétiques et politiques, propres au courant artistique dans lequel s'inscrivent ces effigies, je m'attacherai à la façon dont les marionnettistes les mettent en jeu, sur un mode qui oscille en permanence entre illusion et distanciation, révérence et iconoclasme, précision documentaire et lignes de fuite imaginaires.

\section{De la sculpture à la marionnette hyperréaliste}

Si le désir de mettre en jeu des effigies confondantes de réalisme n'est pas une nouveauté dans le champ de la marionnette ${ }^{2}$, deux phénomènes ont ravivé, ces dernières années, le désir des marionnettistes de travailler dans une perspective, non plus seulement illusionniste, mais "hyperréaliste». Le premier, d'ordre technique, est l'accès aux matériaux de synthèse (silicones, résines polyester, mousses de polyuréthane...) et aux nouvelles possibilités qu'ils offrent en termes de moulage et de sculpture. Le second, d'ordre esthétique, est lié à l'influence des expérimentations conduites par les sculpteurs contemporains ${ }^{3}$.

À strictement parler, la notion d'hyperréalisme, forgée par la critique d'art américaine au début des années 1970, qualifie, si ce n’est un mouvement, en

2. D. Plassard rappelle en effet que « dès le XIX siècle, la multiplication des fils et l'introduction de divers trucages» ont permis aux marionnettistes de faire accomplir à leurs figurines des mouvements toujours proches de ceux des interprètes de chair et d'os, "cette évolution s'accentu[ant] au cours de la seconde moitié du XIX ${ }^{\mathrm{e}}$ siècle, dans les spectacles de marionnettes à fils des compagnies foraines, des music-hall et des théâtres de variétés» (D. Plassard, "Pour une mutation du regard. Marionnettes réalistes, marionnettes hyperréalistes ", Le Point critique, Puck, $\mathrm{n}^{\circ}$ 17, 20IO, p. 3I). Dans son article, D. Plassard rapporte ainsi que, dans les spectacles que présentent à Paris, dans les années I880, les Anglais James et Thomas Holden, la finesse de la construction et la virtuosité de la manipulation des marionnettes étaient telles que les spectateurs avaient le plus grand mal à discerner les acteurs de bois des acteurs vivants. Lors de l'Exposition universelle de 1900, "le Théâtre des Bonhommes Guillaume [...] pousse à son tour très loin l'imitation de personnages vivants, produisant grâce à ses figurines de $50 \mathrm{~cm}$ de haut [- figurines dont les costumes et les perruques sont l'œuvre de célèbres couturiers et coiffeurs -] l'effet d'une société en réduction" (ibid. p. 32).

3. Aussi bien J. Mayer que I. Darras et J. Tenret s'accordent ainsi à revendiquer l'influence de R. Mueck, dont les sculptures hyperréalistes ont été exposées pour la première fois en France à la fondation Cartier (Paris), du I9 novembre 2005 au I9 février 2006. C'est également le cas de B. Vantusso (compagnie Trois Six Trente) qui, depuis quelques années, a développé tout un cycle de création fondé sur la mise en jeu d'effigies hyperréalistes (un enfant dans Kant, de Fosse, des vieillards dans Les Aveugles, de Maeterlinck, des adolescents dans Violet, de Fosse). Le lecteur désireux d'en apprendre davantage sur les créations de la compagnie Trois Six Trente pourra se reporter à l'article de B. Vantusso, «Hyper-marionnettes : les jeux du réel et de l'illusion", J. Sermon (dir.), La Marionnette? Traditions, croisements, décloisonnements, Théâtrel Public, n 193, Gennevilliers, 2009-2, p. 85-87. 


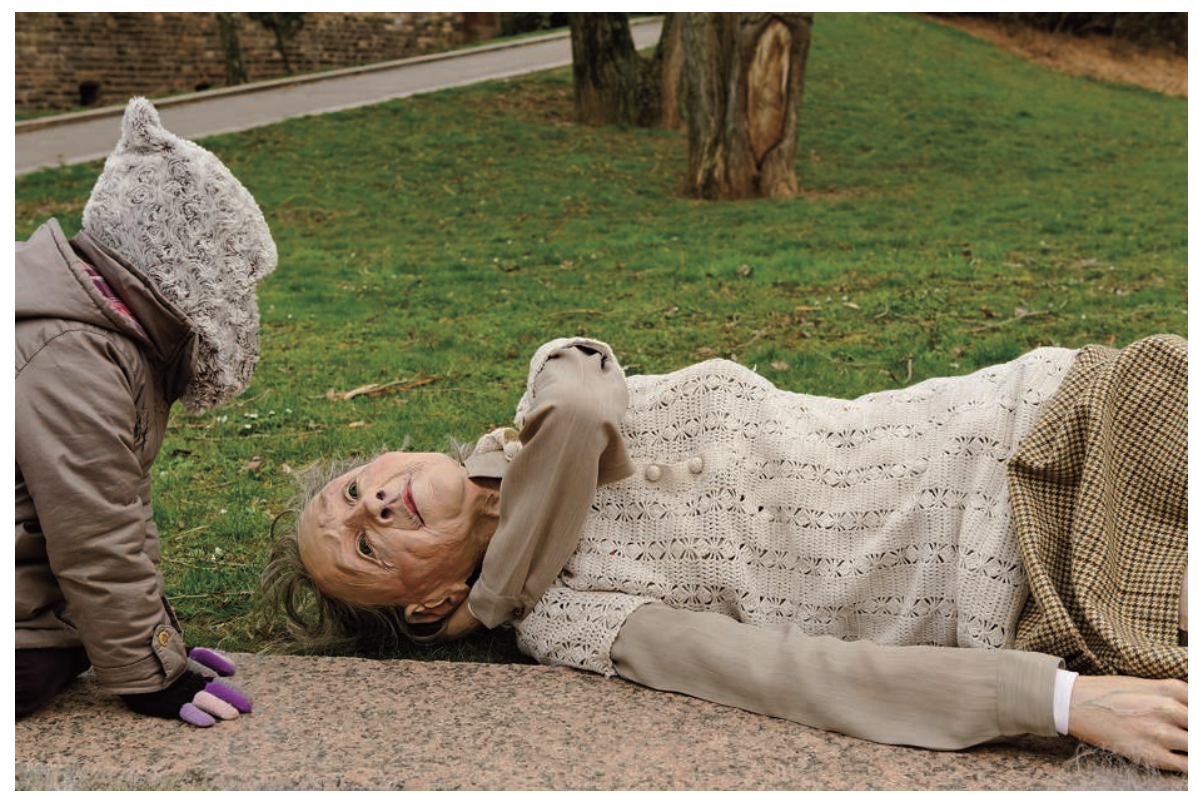

\section{Reprendre son souffle}

Performance urbaine de la compagnie Là Où (Julika Mayer, Renaud Herbin, Paulo Duarte)

Conception Julika Mayer, Rennes, 2009.

\section{(C) Julika Mayer}

tout cas une technique picturale, qui consiste à reproduire le plus rigoureusement possible, non pas la réalité elle-même, mais des images photographiques de cette réalité. Que le peintre ait lui-même pris la photo à partir de laquelle il travaille, ou qu'il l'ait récupérée dans son environnement, son objectif est le même : retranscrire sur la surface de la toile, et avec les outils propres à son médium (couleurs, pinceau, aérographe, couteau à palette...), les qualités et les spécificités de la prise de vue photographique (son grain, sa focale, ses effets de cadrage, de netteté, de flou...) - le tableau abouti produisant, au final, un effet de réel au second degré. Très vite, cette notion d'hyperréalisme a été étendue au champ sculptural, sans faire l'objet d'une élaboration théorique spécifique. Passant des images en à-plat aux images en volume, la question de la surface d'enregistrement - l'empreinte - à partir de laquelle l'artiste élabore son œuvre se pose toutefois différemment : ce n'est plus, en effet, à partir d'une photographie, mais de la technique du lifecasting - soit, des moulages réalisés à partir de modèles vivants ${ }^{4}$ - que Duane Hanson et John de Andrea, figures

4. Cette technique est pour la première fois expérimentée par le sculpteur américain George Segal (1924-2000) qui, au début des années I960, réalise ses premiers "tableaux vivants»- 
pionnières de la sculpture hyperréaliste, réalisèrent leurs œuvres. Si, d'un point de vue politique, les réalisations de ces artistes sont incomparables ${ }^{5}$, leurs techniques de fabrication, elles, sont similaires : le moulage effectué à même le corps humain sert de moule négatif, à partir duquel les sculpteurs tirent un positif, fabriqué en résine polyester et en fibre de verre. La figure obtenue est ensuite peinte par les artistes, qui s'attachent à reproduire dans les moindres détails l'épiderme, la carnation, la pilosité de leurs personnages, qu'ils parent également de chevelure (synthétique ou naturelle), et qu'ils habillent, éventuellement, de véritables vêtements - autant de matériaux, de techniques et d'artifices dont vont s'emparer les marionnettistes contemporains.

Ces dernières décennies, avec l'arrivée d'une nouvelle génération de sculpteurs - tels que Evan Penny (1953-), Ron Mueck (1958-), Sam Jinks (1973-) -, le terme d'hyperréalisme a acquis une extension encore plus large. En effet, loin de se référer à un mode de production particulier (à savoir : des œuvres réalisées, à l'échelle I, selon la technique du lifecasting), l'épithète «hyperréaliste» qualifie désormais des œuvres qui ont une triple spécificité, également à l'œuvre des spectacles de marionnettes auxquels s'attache cet article. La première est de reposer sur un très long, minutieux et patient travail artisanal qui, paradoxalement, vise à reproduire la "parfaite» résolution des images à laquelle en principe seules - et, de surcroît, instantanément - nos prothèses technologiques nous donnent accès. La seconde spécificité de ces œuvres est de susciter un profond trouble perceptif, lié au fait que le très puissant effet de réel qui se dégage des figures sculptées se trouve systématiquement contrebalancé par ce qu'elles peuvent avoir de parfaitement artificiel - que ce soit au niveau de leur taille (réduite ou agrandie), de leur anatomie (plus ou moins anamorphosée), ou de leur mise en situation (étrange, absurde ou fantasque). La troisième, enfin, est d'élargir le champ de nos visibilités, en termes qualitatifs (les figures hyperréalistes sont souvent représentées selon des angles ou à des échelles insolites), mais également, quantitatifs. En effet, au lieu de se cantonner à la reproduction des canons de la beauté sculpturale (au sens artistique, mais

soit : des sculptures directement moulées, par l'entremise de bandes plâtrées, sur des modèles vivants, que l'artiste met en scène et "saisit» dans des postures et des environnements plus ou moins sophistiqués. Son travail n'est toutefois pas considéré, à proprement parler, comme hyperréaliste, dans la mesure où il est impossible de confondre ses sculptures de plâtre avec d'authentiques personnes.

5. Tandis que D. Hanson (I925-1996) entend porter, par le choix des sujets qu'il représente de manière hyperréaliste (retraités floridiens, femmes au foyer, touristes, employés de ménage...), un regard critique sur les stéréotypes, leurres et paradoxes de l'american way of life, le travail de John de Andrea (I94I -) n'a, lui, aucune visée contestatrice : il vise au contraire, dans la parfaite lignée de l'idéal classique, à sublimer la beauté des corps - toujours jeunes, nus, et très majoritairement féminins. 
aussi, sociétal de l'expression), les représentants de la sculpture hyperréaliste placent leurs spectateurs face aux visions, aussi fascinantes que déconcertantes, de corps de tous âges - du nouveau-né au vieillard - et de toutes morphologies - corps obèses, moribonds, de femmes enceintes, et, de façon à la fois plus large et plus simple, corps ordinaires, relevant de l'humanité quelconque ${ }^{6}$, avec ce qu'elle peut avoir d'imparfait, dérisoire, fragile, et de pas forcément séduisant.

Dans cette perspective, il semble logique que les personnes âgées soient un sujet de prédilection pour les sculpteurs contemporains, qui trouvent dans ces corps ridés, aux traits irréguliers, aux chairs avachies, non seulement un matériau plastique de choix, mais aussi, l'occasion de faire converger le regard des spectateurs vers des identités que nos sociétés occidentales - vieillissantes, mais obsédées par la quête de la jeunesse éternelle - tendent à exclure du champ des visibilités. Par l'exposition publique de ces figures présentant les stigmates du vieillissement, les artistes font alors accéder à la dignité de l'espace symbolique ceux et celles qui, sous les effets conjugués des opérations de lissage (chirurgie esthétique, logiciels de retouche d'image) et des dispositifs d'isolement (placement des personnes âgées dans des cercles, clubs, instituts spécialisés...), en sont en principe refoulés.

Si forcer le regard, attirer et renouveler l'attention du spectateur est une chose, choisir de faire des vieillards hyperréalistes les protagonistes d'une représentation marionnettique en est encore une autre. En passant de l'effigie statique à l'effigie animée, en permettant à ces figures, d'une part, d'évoluer dans l'espace, et d'autre part, de s'inscrire dans la temporalité d'une narration, les marionnettistes Julika Mayer, Isabelle Darras et Julie Tenret contribuent à déplacer encore un peu plus les lignes de ce que Jacques Rancière appelle le "partage du sensible». Telle est en tout cas l'hypothèse que je propose d'examiner, en m'attachant à mettre en évidence ce que provoque et ce qu'autorise, aussi bien pour les actrices-manipulatrices que pour les spectateurs, la mise en jeu de marionnettes de vieillards hyperréalistes.

\section{Enjeux symboliques de la manipulation à vue}

Par-delà leurs différences de registre, sur lesquelles je vais revenir, les spectacles de Julika Mayer d'un côté, d'Isabelle Darras et de Julie Tenret de l'autre, ont pour spécificité commune de reposer sur un principe de manipulation à vue. Sur scène, la coexistence des corps de vieillards hyperréalistes et de ceux des

6. Voir G. Agamben, La communauté qui vient. Théorie de la singularité quelconque (trad. M. Raiola), Paris, Seuil, coll. «La librairie du Xxe siècle», I990. 
jeunes femmes (toutes trois sont trentenaires) qui les manipulent, permet de déployer et théâtraliser, non pas seulement l'histoire ou les visions de personnages âgés, mais la géométrie variable des relations qui peuvent se nouer entre eux et celles qui les accompagnent.

Dans Silence, les manipulatrices choisissent de fictionnaliser le rapport qui les lie aux effigies, en s'inscrivant de manière vraisemblable dans le microcosme dramatique : elles jouent le rôle d'aides-soignantes, personnel affecté à la maison de retraite médicalisée où se trouvent les deux protagonistes qu'elles mettent en jeu (un homme, une femme), et vis-à-vis desquels, partant, elles vont tour à tour avoir des fonctions de soin, d'assistance ou de contrôle. Ce faisant, les marionnettistes mobilisent les ressorts symboliques et jouent des analogies qu'on peut établir entre, d'un côté, la poétique de la manipulation (l'illusion de vie de la marionnette dépend de la qualité des mouvements et des énergies qui lui sont impulsés, de l'extérieur, par celui ou celle qui la manipule), et de l'autre, la personne âgée, qu'on aura précisément tendance à qualifier de "vieille» à partir du moment où, perdant totalement ou partiellement son autonomie (motrice, psychique), elle se retrouve en situation de dépendance. Dans Silence, les rapports de pouvoir que cette répartition des rôles semblerait impliquer donnent toutefois lieu à des configurations plus complexes et nuancées : d'une part, parce que les deux aides-soignantes présentent des visages variés (elles sont tour à tour attendries, amusées ou agacées par les vieillards dont elles s'occupent), et d'autre part, parce qu'elles n'endossent en réalité ce rôle que par intermittence. Dans l'essentiel du spectacle, en effet, les deux actrices ne sont «que» manipulatrices : elles se mettent en retrait, faisant oublier leur présence pour se mettre entièrement au service du couple de vieillards - Jean et Élise - qu'elles animent selon la technique de la marionnette portée?

À la différence d'Isabelle Darras et de Julie Tenret, Julika Mayer choisit de ne jamais faire corps avec les effigies hyperréalistes qu'elle met en jeu dans les différentes créations du cycle des Vieilles ${ }^{8}$ : quelle que soit leur taille (réduite

7. On appelle «marionnette portée» une marionnette qui est plaquée contre le corps du marionnettiste (le plus souvent, il la porte contre son buste) et à laquelle il délègue ses propres membres (bras, mains, jambes), introduits à l'intérieur du tronc de la marionnette.

8. Ce cycle de création est constitué de quatre formes : Des nouvelles des vieilles (2007), performance d'une trentaine de minutes pouvant aussi bien se jouer en salle qu'en extérieur, et qui prend la forme d'un duo chorégraphique entre J. Mayer et une marionnette de vieille femme hyperréaliste mesurant environ I,20 m; Reprendre son souffle (2009), performance improvisée dans l'espace urbain par les trois membres de la compagnie Là Où (J. Mayer, R. Herbin, P. Duarte), qui accompagnent et mettent en jeu des marionnettes de vieilles hyperréalistes de tailles diverses (entre $30 \mathrm{~cm}$ et $\mathrm{I}, 8 \mathrm{o} \mathrm{m}$ ); Ritournelle (20IO), spectacle en salle d'environ une heure, qui fait dialoguer trois âges, trois langages et trois présences : J. Mayer, marionnettiste trentenaire, 


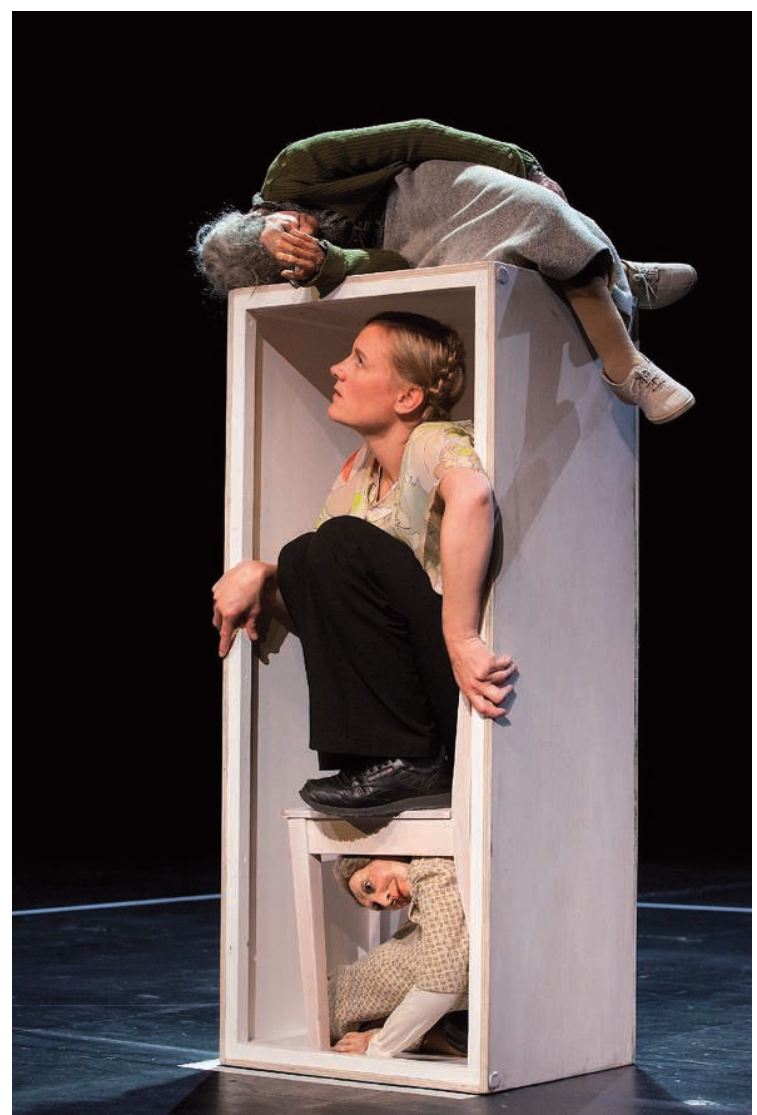

\section{Ritournelle}

Spectacle mis en scène par Julika Mayer Interprété par Julika Mayer et Marie-Joséphine Thomas Rennes, 20IO.

(C) Julika Mayer

à celle d'une poupée ou pouvant atteindre plus de deux mètres), ce sont des corps intégraux et extérieurs à elle que la marionnettiste manipule, le plus souvent en prise directe, ou via une petite crosse placée dans le dos des marionnettes. De surcroît, même dans Ritournelle, qui est la plus théâtrale des différentes formes composant le cycle, il est impossible de parler d'un quelconque microcosme dramatique : s'il y a bien des effets de fiction, des figures

M.-J. Thomas, chanteuse lyrique sexagénaire, les effigies de vieilles femmes hyperréalistes; En plis (20IO), installation sonore et visuelle dans laquelle trois proches collaborateurs de J. Mayer (le plasticien P. Duarte, le vidéaste N. Lelièvre, et le musicien M. Daguenet) déploient et interprètent les divers matériaux, documentaires et artistiques, collectés au cours du cycle de création. 
et des situations reconnaissables, ceux-ci, d'une part, sont toujours fugaces, et d'autre part, naissent de manipulations qui sont moins d'ordre théâtral que chorégraphique et installatif. Chorégraphique, dans le sens où Julika Mayer, qui s'est formée à diverses techniques corporelles (danse contact-improvisation, yoga, Feldenkrais...) après sa sortie de l'École nationale supérieure des arts de la marionnette (ENSAM), cherche moins à animer la marionnette (c'est-à-dire, à lui impulser un mouvement chargé d'expressivité, d'intentionnalité, et donc, pourvoyeur d'illusion de vie), qu’à explorer la façon dont son propre corps peut entrer en contact et en dialogue avec les propriétés physiques de l'effigie (son poids, ses articulations, sa souplesse, sa résistance). Installatif, dans le sens où Julika Mayer, non contente de mettre en mouvement la marionnette selon des modes qui ne sont plus prioritairement narratifs ou affectés de subjectivité, s'autorise à régulièrement ramener les effigies hyperréalistes à leur dimension sculpturale, en les plaçant, assises ou allongées, sur différents objets (chaise, table, caisson) qui leur tiennent lieu de piédestal; si l'on peut alors encore parler de manipulation, c'est au sens littéral du terme - "action de toucher, tenir, transporter avec les mains".

À défaut de raconter une histoire en particulier et de donner consistance, de manière cohérente et durable, à des identités dramatiques, les jeux de corps à corps, de regards, de déplacements et de placements auxquels Julika Mayer livre ses effigies, permettent toutefois d'esquisser une multitude de relations et de figures possibles. Selon que la marionnettiste se tient au-dessus, en dessous, derrière ou à côté de la marionnette, selon qu'elle la porte contre son ventre, sur son dos, ses épaules, ses genoux, ou qu'elle pose sa propre tête au creux du giron de l'effigie, selon qu'elle l'accompagne précautionneusement dans ses mouvements ou décide au contraire de la faire vivement tournoyer, ce qui s'offre au jeu des projections imaginaires du spectateur, ce sont non seulement les images de la vieillesse dégradée (la vieille femme assistée par une plus jeune qu'elle) ou de la vieillesse paisible (la grand-mère réconfortant sa petite-fille), mais aussi, celle de la mère guidant son enfant, ou celle de l'enfant jouant avec sa poupée, ou encore, celle de la marionnettiste trentenaire portant, à la manière de La Classe morte de Kantor, ses doubles vieillis. Au trouble ontologique qui caractérise les marionnettes en général (aux confins de l'animé et de l'inanimé, du matériel et du spirituel) et la marionnette hyperréaliste en particulier (rendant confuse la perception des frontières entre la vie et l'art, le réel et l'artificiel'), la façon qu'a Julika Mayer de mettre en jeu ses effigies en

9. À ce titre, D. Plassard note que si les «figures [hyperréalistes] sont d'une étrangeté si saisissante que l'on est d'abord tenté de les rapprocher du "théâtre d'andrö̈des" dont rêvait Maeterlinck", une différence s'impose toutefois. En effet, si ces créatures artificielles ont bien, 
adjoint ainsi un autre : celui qui naît, non pas d'un entre-deux, mais des effets d'instabilité et de réversibilité qui se trouvent instaurés entre deux positions - jeunesse / vieillesse - a priori bien étanches et établies.

\section{Réalisme, déréalisation, étonnement}

Dans un article qu'il consacre à la question du réalisme et de l'hyperréalisme dans les théâtres de marionnettes, Didier Plassard note que le choix de travailler avec des effigies hyperréalistes provoque "un renversement des protocoles traditionnels de jeu ${ }^{\mathrm{IO}}$ ». La première raison en est que, "dotée par le sculpteur d'une qualité de présence exceptionnelle, la marionnette n'a plus besoin d'être animée pour donner le sentiment de la vie, et c'est plutôt comme un témoin, un soigneur, un parent que le marionnettiste peut s'approcher d'elle» - autant de «figures d'accompagnement" particulièrement bien adaptées à la mise en jeu de personnages de vieillards. Simultanément - et c'est le deuxième changement notable - le manipulateur, «en partie dégagé de la responsabilité de donner vie à la marionnette", va "se trouve[r] en charge d'autres fonctions, et principalement de celle de rethéâtraliser la représentation en faisant apparaître sa dimension de simulacre» : "face au pouvoir de sidération de la sculpture hyperréaliste», l'acteur-manipulateur tend alors à se faire "opérateur de déréalisation ». À l'appui de cette analyse, Didier Plassard mentionne un certain nombre d'exemples (dont le spectacle de Julika Mayer, Les Nouvelles de vieilles) où, par des formes de manipulation diversement invraisemblables ou brutales, les marionnettistes s'appliquent à «rend[re] à la figure [hyperréaliste] son statut d'objet pour rompre l'empathie née de sa ressemblance avec le vivant». Aussi juste soit-elle, cette analyse me paraît, dans la perspective générale du questionnement qui est le nôtre (la représentation de la vieillesse) et, celle, particulière, des choix de manipulation propres à Silence, appeler quelques nuances.

Ainsi, dans le cycle des Vieilles, il est indéniable que la légèreté voire la désinvolture avec lesquelles Julika Mayer peut manipuler ses effigies (qu'elle fait sauter ou onduler dans les airs, tourner autour d'elle-même en les tenant par les jambes, qu' elle traîne au sol en les tirant par un bras...) contreviennent

comme les figures de cire qui fascinaient Maeterlinck, «les allures de la vie sans avoir la vie» ( Menus propos - Le théâtre», I890), elles sont en revanche loin de provoquer l'effroi que suscitent "les figures de cire, avec leurs reflets de chair morte»; elles ont au contraire " toutes les qualités visibles d'une chair vivante» (D. Plassard, «Pour une mutation du regard. Marionnettes réalistes, marionnettes hyperréalistes", art. cité, p. 39).

Io. Ibid., p. 40. Les citations qui suivent dans le paragraphe sont également extraites de cette page. 


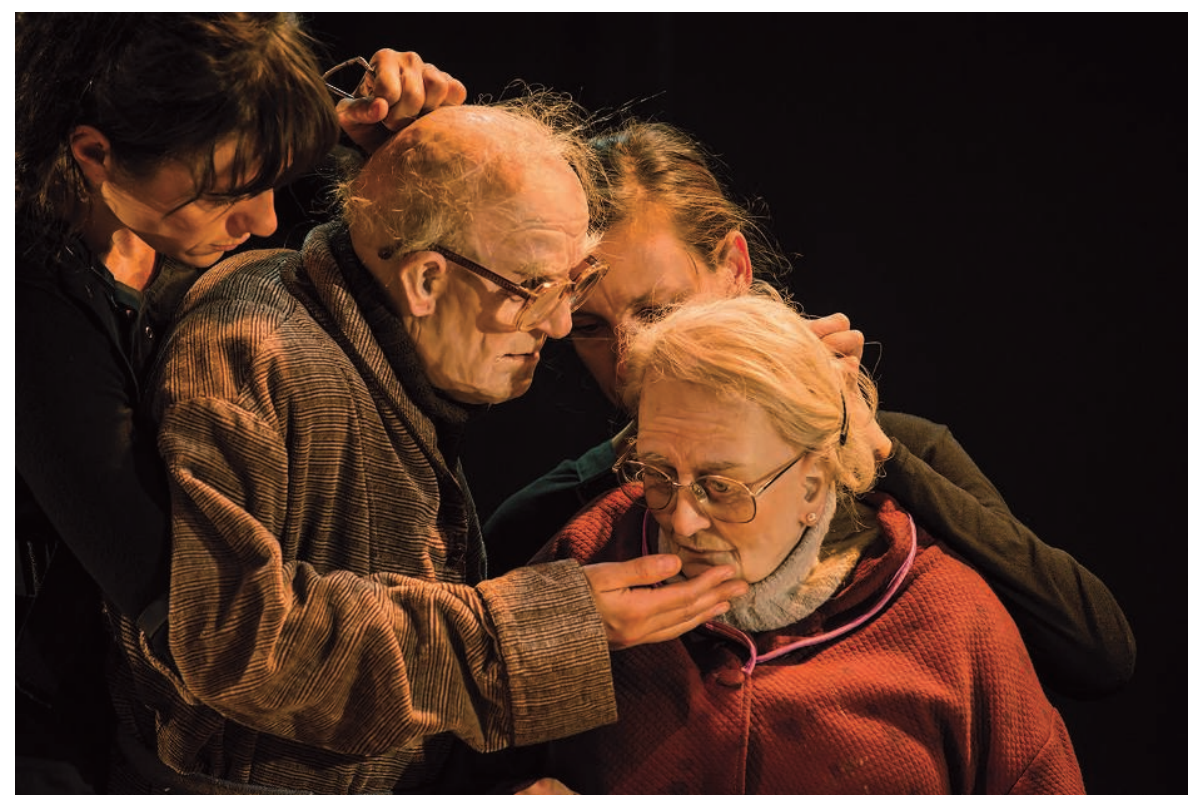

\section{Silence}

Spectacle interprété par Isabelle Darras et Julie Tenret

Mis en scène par Bernard Senny, Compagnie Night Shop Théâtre, Bruxelles, 2013.

(C) Yves Kerstius

aux règles de la vraisemblance et de la bienséance, et donc, interrompent les phénomènes d'identification. Cela dit, cette mise à distance de la gravité - au sens physique et moral du terme - doit aussi être mise en relation avec le projet documentaire de la marionnettiste, dont le cycle de création s'adosse à un ensemble d'entretiens que Julika Mayer a réalisés, dans différentes villes d'Europe, avec des "vieilles» qu' elle est allée rencontrer pour les faire parler, non seulement de leur rapport à la fatigue, à la maladie, à la mort, mais aussi, à la sexualité, à la gourmandise, à tout ce qui, dans leur présent et de leur point de vue de vieilles femmes, a trait aux plaisirs de la vie. De la collection de témoignages qu'elle a pu rassembler, Julika Mayer prélève et donne à entendre, au gré des créations, des extraits aux tonalités variées, tant du point de vue de ce qui s'y raconte (les femmes sont tour à tour joyeuses, drôles, résignées, mutines, sévères...) que de celui du timbre, du souffle et de l'intensité propres aux voix qui s'y expriment. Diffusés via une bande-son, ces inserts audio viennent, dans chacune des pièces du cycle, ponctuellement s'entrelacer aux images, arrêtées ou en mouvement, que composent les interprètes et les effigies - avec tous les jeux de contrepoint, d'écho, de concordance ou de discordance qui peuvent s'opérer, pour le spectateur, entre les plans sonore et visuel. 
Dans Ritournelle, où, comme dans les autres spectacles du cycle, des fragments d'enregistrement sont restitués, Julika Mayer choisit, en outre, de reprendre certaines des questions qu'elles avaient pu poser dans le cadre de ses entretiens, et de les adresser directement à la chanteuse lyrique sexagénaire (Marie-Joséphine Thomas) qui l'accompagne pour l'occasion. Cette dernière, que Julika Mayer avait d'abord rencontrée dans le cadre du projet d'entretiens qu'elle conduisait, avait confié à la marionnettiste que les cantatrices sont confrontées à deux morts : celle de la voix, puis la leur. Partant de là, Julika Mayer a souhaité poursuivre l'aventure de l'entretien, et a proposé à Marie-Joséphine Thomas de l'accompagner sur le plateau, où elle jouerait son propre rôle (une interprète de soixante ans), mais aussi, un rôle de fiction (la femme octogénaire qu'elle s'imagine devenir). Né de la rencontre entre ces "deux femmes : une jeune, une vieille ${ }^{\mathrm{II}} »$, Ritournelle prend alors la forme d'un jeu de miroirs à plusieurs fonds, où : «Une jeune met en scène la grande vieillesse encore fictive d'une vieille pas si vieille. / Une jeune plus si jeune interroge une vieille pas si vieille : elles se "parlent" avec leurs langages respectifs : les marionnettes et les objets pour l'une, le chant et le théâtre pour l'autre. [...] L'une anime les marionnettes : tentative vaine mais propre à toute manipulation : "ramener à la vie", se faire face, se dédoubler, se projeter dans l'autre, se projeter dans le temps. / L'autre joue et déjoue la fiction de sa propre vieillesse. » À deux reprises au cours du spectacle, la «jeune» choisit toutefois d'abandonner son médium de prédilection pour interpeller directement "la vieille». À travers les questions qu' elle lui pose - par exemple : «Qu'est-ce qui te fait lever le matin?»; "Est-ce qu'avec l'âge, tu ressembles à ta mère?»; "Tu as froid? "; "Qu' est-ce que votre génération de femmes a fait pour la mienne?» "Comment tu te sens femme aujourd'hui?»; "Qui va te sécher les cheveux quand tu auras quatre-vingt-quinze ans?; "Combien d'âges as-tu?» - il apparaît alors clairement que, tant du point de vue des images qu' elle donne à voir que des propos qu'elle donne à entendre dans son cycle de création, le projet de Julika Mayer est moins de représenter l'état de vieillesse que de questionner l'identité des femmes dans leur devenir, à travers et par-delà la segmentation sociale des "classes d'âge».

Dans Silence, où, comme dans les spectacles de Julika Mayer, les seules et rares paroles prononcées le sont, non pas par les marionnettes, mais par les interprètes qui les accompagnent, c'est également à travers une série de questions que va se dessiner un premier portrait de la vieillesse. Assises face au public, et revêtues de leur blouse d'aides-soignantes, Isabelle Darras et Julie Tenret vont en

II. Ici et dans la suite du paragraphe, les expressions citées entre guillemets sont extraites du texte de présentation de Ritournelle que J. Mayer a rédigé pour le dossier artistique des Vieilles. 
effet à deux reprises (en ouverture, puis aux environs de la moitié du spectacle) jouer à une partie de Qui est-ce? ${ }^{\text {r2 }}$. Si les premières questions, qui portent sur l'apparence physique des personnages ("Est-ce qu'elle a des cheveux blancs?», «Est-ce qu'il a encore des cheveux?», "Est-ce qu'elle a des lunettes?»), sont conformes aux règles de ce jeu, elles y dérogent toutefois assez vite. À partir du moment, en effet, où les interrogations portent, non plus sur la physionomie de la personne à identifier, mais sur son caractère («Est-ce qu'il est plutôt doux ou têtu?», "Est-ce qu'elle est difficile?»), ou encore, ses comportements et modes de vie («Est-ce qu'il se lave tout seul?», «Est-ce qu'elle a des visites?», «Est-ce qu'elle nous reconnaît?»), il est a priori impossible, à la simple vue des portraits que présentent les cartes à jouer, de répondre à ces questions. C'est pourtant bel et bien ce que font les deux actrices, qui continuent d'avancer dans la partie et défaussent peu à peu les cartes de ceux ou celles qui ne concordent pas à la description - le nombre de volets rabattus contre le plateau de jeu, à chaque phase d'élimination, étant source d'effets comiques, mais aussi, parfois plus pathétiques. Ainsi, le fait d'apprendre que, oui, "elle regarde Les Feux de l'amour», ou que non, on ne l'a jamais "retrouvé tout nu dans le jardin", permettra, à chaque fois, de n'éliminer qu'une seule carte. À l'inverse, onze volets seront rabattus d'un coup lorsqu'on apprendra que l'identité mystère est «toujours vivante». Par le détournement comique et ludique auquel elles livrent le jeu Qui est-ce?, Isabelle Darras et Julie Tenret s'autorisent ainsi, en ouverture du spectacle, à traverser la plupart des stéréotypes et des angoisses que l'on attache à la vieillesse (appauvrissement intellectuel et affectif, perte de son intégrité physique et psychique, maladie, mort).

S’il s'agit là de réalités indéniables, les deux artistes ne s'en satisfont toutefois pas et, prenant le contrepied des visions globalement sombres et négatives évoquées en préambule, elles vont s'attacher à éclairer sous un autre jour la vie des personnes âgées - et ce, dès la séquence qui succède immédiatement à la partie de Qui est-ce? Ayant rangé leurs plateaux de jeu, les manipulatrices reviennent en effet avec un petit chariot transportant un groupe de huit pantins $^{13}$ (six femmes, deux hommes), qu'elles vont installer un à un sur la table de manipulation. Si, par leurs têtes (faites d'un portrait photographique découpé et rattaché au corps par un petit ressort) et par leurs costumes (faits

I2. Édité pour la première fois en France en 1979, ce jeu de société pour deux joueurs consiste à deviner, par une série de questions et de déductions, quelle est l'identité de la carte choisie par l'adversaire parmi les 24 portraits que donne à voir chacun des deux plateaux de jeu.

I3. "Pantin» est à entendre au sens strict du terme : "Figurine de carton ou de bois plat et mince, découpé et colorié, représentant généralement un personnage burlesque, et dont les membres articulés sont actionnés au moyen d'un fil» (Trésor de la langue française). 
d'authentiques matériaux ${ }^{14}$ ), ces effigies peuvent annoncer celles, hyperréalistes, qui entreront plus tard en scène, elles se distinguent cependant radicalement de ces dernières, tant leur taille (variant entre 30 et 45 centimètres), la stylisation de leur anatomie (tête disproportionnée par rapport au corps, bras tout petits et schématiques) et les qualités de leurs mouvements (saccadés, brusques, tremblotants) les ramènent à leur statut d'artéfacts. Pour ne pas reposer sur un principe d'illusion réaliste, la scène à laquelle ces figurines prennent part ne s'en trouve pas moins chargée de signification. En effet, en choisissant de faire défiler puis danser les pantins, en jouant, non seulement de toutes les vitesses de déplacement (très ralentie ou au contraire accélérée), mais aussi, des propriétés (mécaniques, cinétiques) propres à leur facture, les marionnettistes opposent, à la vision morne, uniforme, que connote le terme générique de «vieillesse», le spectacle animé d'un groupe de vieillards aux énergies disparates, aux silhouettes contrastées et à l'allure bigarrée.

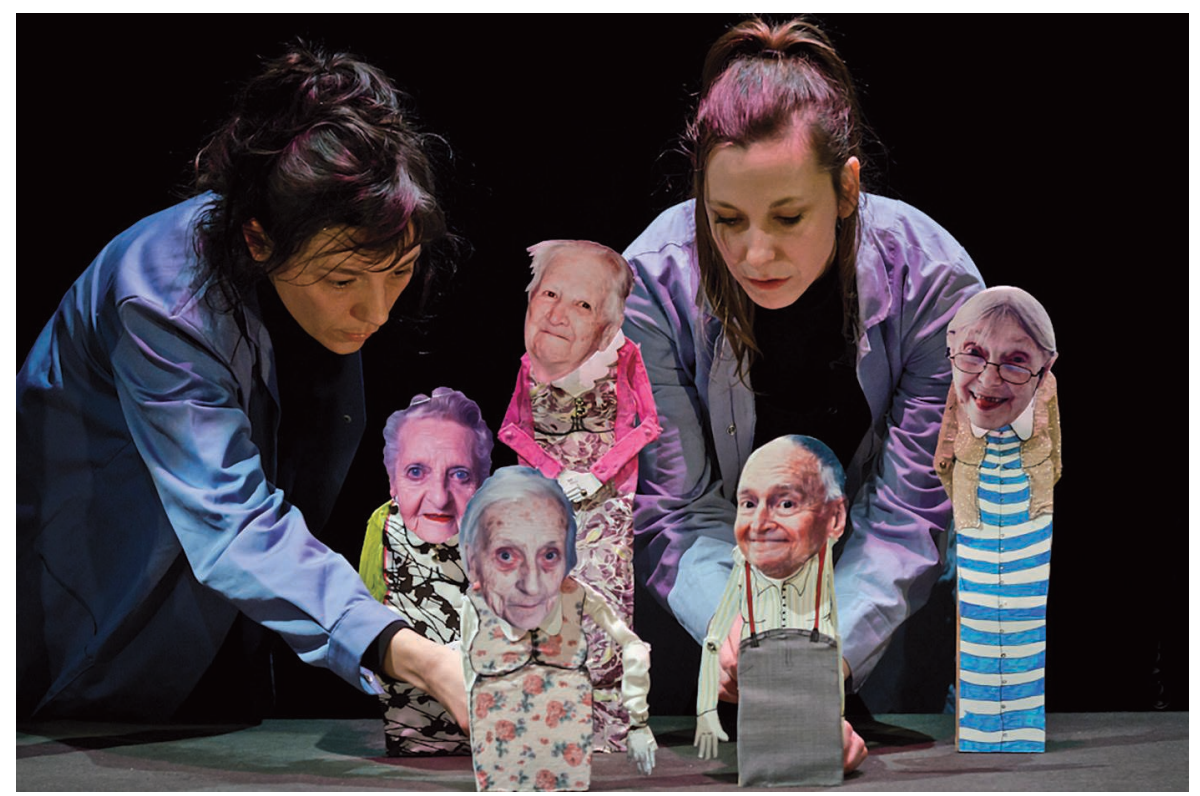

\section{Silence}

Spectacle interprété par Isabelle Darras et Julie Tenret Mis en scène par Bernard Senny, Compagnie Night Shop Théâtre, Bruxelles, 20I3.

(C) Yves Kerstius

14. Les marionnettistes de Silence ont en effet choisi, non pas de colorier le corps de leurs pantins (voir note précédente), mais de leur fabriquer de petits costumes en tissus, qui sont accolés sur la surface des silhouettes. 
Avec l'arrivée des deux marionnettes hyperréalistes, le registre presque forain qui caractérisait la précédente séquence, fait place à une tonalité et à un espace beaucoup plus intimistes. Prenant place au milieu du mobilier (une armoire, une commode, une table, deux chaises, divers bibelots) qui structurent l'intérieur de Jean et Élise, les manipulatrices s'effacent alors pour se mettre au service du couple que forment ces deux personnages - personnages qu'elles animent avec une précision proprement naturaliste. En effet, si la technique de la marionnette portée permet, en tant que telle, de doter les mouvements de la marionnette de toute la fluidité et spontanéité propres au corps vivant, Isabelle Darras et Julie Tenret ont, dans ce spectacle, cherché à atteindre la plus grande vérité de jeu possible et, désireuses de trouver les attitudes, les gestes et les mimiques les plus justes et évocateurs qui soient, ont nourri leur travail d'animation de l'observation de personnes âgées (à commencer par leurs propres grands-parents). Couplée à la puissance expressive des visages hyperréalistes, cette attention portée à la qualité des mouvements (gestuelle et déplacements) fait que le sentiment de vie qui se dégage des effigies atteint des points absolument saisissants.

Si impressionnant soit-il, cet art de la manipulation ne serait toutefois qu'un exercice de virtuosité si les marionnettistes ne s'employaient pas à le mettre au service d'un projet qui entend affronter certains tabous et montrer la vieillesse sous un jour inaccoutumé. De fait, ce que les saynètes muettes de Silence donnent à voir, c'est le quotidien d'un couple âgé, et plus précisément, les gestes de tendresse, les petites attentions, les moments de complicité mais aussi, les moments de perdition qui trament leur vie dès lors que l'un d'eux - en l'occurrence : la femme - se trouve atteint de la maladie d'Alzheimer. À la différence, toutefois, de Michael Haneke qui, dans Amour (20I2), explore très crûment la façon dont l'union d'un vieux couple se trouve mise à l'épreuve de la dégénérescence physique et mentale, Isabelle Darras et Julie Tenret, elles, demeurent pudiques, préférant toujours la suggestion à la monstration. De surcroît, autant le réalisateur se sert de sa caméra comme d'un œil clinique, autant les marionnettistes vont choisir de transférer à leurs personnages l'esprit d'enfance qui, d'une manière ou d'une autre, caractérise toujours ceux qui font (ou assistent à) des spectacles de marionnettes. C'est ainsi qu'au cours du spectacle, on verra Élise aller chercher le portrait d'une jeune femme (peut-être le sien, se dit-on ${ }^{\text {Is }}$ ) qui, une fois que le cadre en aura été retiré, s'avère une boîte remplie de gaufres que le couple soustrait, par cet artifice, à la vigilance des

I5. Il est probable que cette confusion n'opère pas auprès du public belge : le jeune visage féminin figurant sur ce qui s'avérera être une boîte à biscuits est celui de la reine Fabiola, reine de Belgique de 1960 à 1993. 
aides-soignantes; ou bien, Jean en train de s'appliquer, dès que sa femme a les yeux tournés, à plier sous la table un journal à partir duquel il lui fabrique un origami d'oiseau; ou encore, Jean pointer son doigt sur le châle d'Élise pour, au moment où elle relève la tête, lui faire une pichenette sur le nez - l'intérêt étant que tous ces petits moments de ruse, de blague, de tendresse, auxquels le spectateur ne s'attend a priori pas, lui parviennent bel et bien avec la charge réaliste et la puissance de conviction qui caractérisent ces effigies et leur mise en jeu.

En conclusion de quoi il me semble possible d'affirmer que, par-delà ce qu'ils peuvent avoir de radicalement différent d'un point de vue esthétique, les spectacles de Julika Mayer d'un côté, d'Isabelle Darras et de Julie Tenret de l'autre, présentent deux intérêts communs. Le premier est d'échapper aux visions monolithiques de la vieillesse (tantôt dépréciée en bloc comme incarnation fatale de la déchéance, tantôt abstraitement idéalisée et subsumée sous la figure de la sagesse) pour proposer un accès singulier, subjectif, à ce que peuvent être la vie et le vécu des vieux. Le second est de profiter des conventions de la marionnette pour décaler nos perceptions, créer des espaces de doute et d'étonnement, et peut-être, élargir l'horizon de nos représentations. 